\title{
Brucelosis humana: situación epidemiológica en Chile, 2001-2010
}

\author{
Paulina Martínez
}

\section{Human brucellosis: epidemiological situation in Chile, 2001-2010}

Background: Brucellosis is a zoonosis whose economical impact in endemic countries is significant. Its magnitude for humans has been scarcely measured in Chile, therefore brucellosis was declared a reportable disease since 2004. Objective: To characterize the epidemiology of human brucellosis in Chile. Material: Data sources were the Obligatory Disease Notification System (ODNS), the National Reference Laboratory for brucellosis, hospital discharges and deaths statistics. Results: The average incidence, according to the ODNS was 0.55 cases per 100.000 inhabitants (men 67.8\%, median age 44 years). Hospital discharges rate of brucellosis during this period were 0.43 per 100.000 inhabitants. The major diagnosis (84.3\%) was A23.9 (Brucellosis unspecified). Two patients died (mortality rate for the period 0.01 deaths per 100000 inhabitants), their education level was basic. Conclusions: The incidence rates of cases reported, hospital discharges and mortality tend to decrease. The discrepancy between reported cases and clinical/laboratory diagnosis suggests underreporting. It is necessary to improve the notification system and to optimize laboratory confirmation by the National Reference Laboratory.

Key words: Brucellosis, human brucellosis, cases reported, hospital discharges, deaths.

Palabras clave: Brucelosis, brucelosis humana, casos notificados; egresos hospitalarios; defunciones.

\section{Introducción}

$\mathrm{L}$ a brucelosis es una zoonosis causada por bacterias del género Brucella, ampliamente distribuida en el mundo, excepto donde está erradicada la brucelosis bovina (B. abortus). La incidencia de la enfermedad es desconocida y la estimación de su carga de enfermedad asociada varía fuertemente desde valores menores a 0,03 hasta 160 casos o más por $100.000^{1}$.

Es una enfermedad que genera alto impacto socioeconómico, tanto por los costos en salud pública (costos de diagnóstico, tratamiento, recuperación y por ausentismo laboral) como por pérdidas en la producción animal (disminución de la producción de leche, concepción y abortos). Por lo anterior, el Ministerio de Salud de Chile (MINSAL) incorporó esta enfermedad al sistema de vigilancia de notificación obligatoria con el objetivo de caracterizar epidemiológicamente los casos, forma de presentación y fuentes de infección, de manera de interrumpir la transmisión y desarrollar medidas de prevención y control en humanos ${ }^{2}$.

El presente trabajo presenta las características generales de la enfermedad y los principales indicadores de esta enfermedad en Chile, según el Sistema de Notificación Obligatoria (ENO), diagnóstico de laboratorio (Instituto de Salud Pública-ISP) egresos hospitalarios, y defunciones oficiales del Registro Civil.

\section{Características generales}

Brucella spp es un cocobacilo, intracelular facultativo, gramnegativo, no capsulado y no formador de esporas. Se han reconocido diez especies de Brucella basadas sobre la especificidad del hospedero preferencial, ocho de las cuales afectan a animales terrestres (B. abortus, B. melitensis, B. suis, B. ovis, B. canis, B. neotomae, B. microti y $B$. inopinata) y dos que afectan a mamíferos marinos $(B$. ceti y $B$. pinnipedialis). A la vez, se han clasificado como Brucellas clásicas a B. abortus, B. melitensis y B. suis; para estas bacterias se han reconocido 7,3 y 5 biovars, respectivamente ${ }^{1,3}$.

De las diez especies reconocidas mundialmente, seis son capaces de infectar al ser humano; según su mayor poder patogénico e invasor, se ordenan en forma descendente como B. melitensis, B. suis, B. abortus, B.canis, B. ceti y $B$. inopinata. Además, debido a su alta naturaleza infecciosa, y dada su facilidad en generar micro-aerosoles, B. melitensis, B. suis y B. abortus son consideradas como potenciales armas biológicas ${ }^{1,3-5}$.

Estas bacterias pueden permanecer viables durante meses en el agua, productos animales (fecas, productos abortados, lana) y materiales de trabajo, incluyendo ropa, siempre que se mantengan condiciones de humedad alta, temperatura baja y poca luz solar. También pueden resistir en el polvo y suelo manteniéndose en condiciones de desecación, especialmente en presencia de material orgánico ${ }^{4}$.
Universidad Diego Portales. Santiago. Chile.

El estudio se realizó sin aporte económico o becas.

La autora declara no tener relación financiera o personal con personas $u$ organizaciones que pudiesen dar lugar a conflicto de interés con este artículo.

Recibido: 28 de enero de 2013 Aceptado: 28 de octubre de 2013

Correspondencia a: Paulina Martínez Gallegos paulinamartinezg@yahoo.com 
La infección se puede transmitir entre animales mediante ingestión, contacto por membranas mucosas o piel con pérdida de continuidad, aunque se sospecha que también mediante piel íntegra, con placentas, líquidos fetales o descargas uterinas/vaginales de un animal infectado. Además, los animales infectados pueden eliminar bacterias después de un parto o aborto; incluso se pueden transformar en portadores crónicos, y continuarían eliminando el microorganismo, tanto por secreciones uterinas como mediante la leche. Por ende, existe trasmisión vertical y horizontal ${ }^{4,6}$.

La enfermedad es transmitida a los humanos, con mayor frecuencia, mediante el consumo de productos lácteos no pasteurizados o por contacto con animales infectados, placentas o productos de abortos ${ }^{7,8}$.

Cuando es observada según grupos poblacionales, es posible establecer una mayor frecuencia de brucelosis en ciertos grupos laborales, particularmente personal con trato habitual con animales, siendo considerada una enfermedad ocupacional ${ }^{9}$; no obstante, la forma de mayor contagio es mediante el consumo de alimentos contaminados ${ }^{7,8}$.

El período de incubación habitual varía entre 1 y 3 a 4 semanas $^{1,8}$, siendo su presentación clínica en forma aguda y sub-aguda. Como infección sistémica, se describe un amplio espectro de síntomas clínicos, usualmente con fiebre ondulante, sudoración nocturna, escalofríos, cefalea, anorexia, artralgias y malestar general. También se pueden registrar variadas complicaciones, entre ellas neurobrucelosis (encefalitis, meningitis, meningo-encefalitis, neuropatía periférica, radiculopatía y anormalidad del comportamiento $)^{10}$, espondilitis, uveítis, artritis, orquitis y prostatitis ${ }^{11}$.

En mujeres embarazadas se pueden producir abortos, generalmente entre el primero y segundo trimestre de gestación. De rara presentación $(<2 \%)$, la complicación de mayor gravedad -incluso fatal-, es la endocarditis, la que está asociada principalmente a infección por $B$. melitensis ${ }^{1,8}$.

Existen muchas similitudes entre el cuadro clínico en las personas y el animal; en ambos hospederos la bacteria se acantona en el sistema retículo-endotelial, incluyendo bazo, hígado, nódulos linfáticos y médula ósea. Además, las bacterias tienen como blanco las células del sistema esquelético y del tracto reproductivo masculino. En ausencia de tratamiento antimicrobiano el microorganismo suele persistir por meses como pequeños granulomas en estos tejidos blanco, causando una importante morbilidad y mortalidad, y eventualmente septicemia ${ }^{8,12}$. Por otra parte, la infección también puede ser asintomática y detectada sólo por evidencia serológica ${ }^{13}$.

La falta de diagnóstico precoz, y por lo tanto un tratamiento apropiado durante la fase aguda, puede generar una difusión bacteriana hacia varios tejidos y órganos y producir una enfermedad sub-aguda o crónica, de difícil tratamiento ${ }^{1}$. Sin embargo, la letalidad es baja y entre quienes no reciben tratamiento se describe una mortalidad entre 2 y $5 \%{ }^{6}$.

El diagnóstico de la brucelosis humana puede ser postergado debido a que la enfermedad principalmente se presenta como fiebre de origen desconocido con signos y síntomas inespecíficos. El aislamiento bacteriano en la sangre, médula ósea, tejidos o fluidos corporales confirma la infección, pero el cultivo tradicional es de bajo rendimiento, especialmente en los cursos crónicos de la enfermedad. En general se menciona que la tasa de aislamiento de la bacteria en sangre es baja, y por lo tanto el diagnóstico de laboratorio se basa principalmente en pruebas serológicas y moleculares ${ }^{14,15}$.

Según la Organización Mundial de la Salud (OMS) el tratamiento de elección es el uso de doxiciclina/ rifampicina ${ }^{16}$.

Finalmente, se ha de considerar que la prevención más eficiente es mediante el control y eliminación de la brucelosis animal; sin embargo, la eliminación es de difícil consecución dada la persistencia en animales silvestres y la persistencia ambiental ${ }^{14}$. Por lo tanto, para prevenir la brucelosis humana se ha de evitar el consumo de productos lácteos no pasteurizados y en grupos ocupacionales, profundizar las medidas educativas y el uso de ropa protectora ${ }^{16}$.

\section{Situación epidemiológica}

Se trata de una enfermedad de importancia en salud pública. Diversas causas modifican la dinámica de su transmisión, como la facilidad de desplazamiento, producto del intercambio comercial ${ }^{17}$.

La brucelosis humana, al igual que otras zoonosis, es una enfermedad de elevados costos socioeconómicos, por concepto de ausentismo laboral, hospitalización, tratamientos médicos, exámenes, consultas médicas especializadas y rehabilitación, entre otras.

Su distribución es mundial pero está controlada en la mayoría de países desarrollados. Por el contrario, la enfermedad clínica aún es común en regiones de Asia, África, América Central y Sudamérica ${ }^{6}$.

Las especies de Brucella prevalentes varían según la zona geográfica pero $B$. abortus se encuentra en todas las regiones ganaderas, salvo en las zonas donde ha sido erradicada como Japón, Canadá, Australia, Nueva Zelandia, Gran Bretaña, países del Norte de Europa e Israel. En mamíferos marinos de prácticamente todas las costas se han encontrado cultivos positivos o serología positiva $^{6}$. En Latinoamérica, Uruguay es el país con menor prevalencia $^{18}$.

En Chile la brucelosis fue descrita serológicamente en 
1930 y el Ministerio de Agricultura abordó este problema a partir del año 1945. Sin embargo, una planificación sistemática para controlar la enfermedad en ganado bovino sólo se inició en el año 1974 con la elaboración del "Proyecto de Salud Animal", donde en paralelo al plan de erradicación de la fiebre aftosa se comenzó el control de la brucelosis bovina ${ }^{19}$. La prevalencia animal entre los años 1976 y 1982 disminuyó de 7 a 2,9\% alcanzando en seis años la meta propuesta por el Proyecto. Sin embargo, en los nueve años siguientes la disminución de la prevalencia ha sido en torno a 0,5\%. En el año 1991, un estudio mostró prevalencia predial nacional de $20,8 \%$, con máximos en la Región Metropolitana (34,4\%) y en la Región de Los Lagos (26,8\%), siendo ambas similares a lo estimado en la medición del año $1976^{19}$.

El proceso de erradicación de brucelosis bovina se inició gradualmente en Chile, entre los años 1991 y 1996. En el año 2003, la Región de Magallanes fue declarada libre mediante campañas de vacunación; en esa región se efectúa vigilancia pasiva (mataderos y en las lecherías de temporada) y en los predios fronterizos con Argentina -dado el riesgo de contacto directo con animales de zonas no libres-, se mantiene la vacunación con cepa RB51. Durante el año 2005 se emitió la resolución que declara también erradicada la brucelosis bovina en Región de Aysén ${ }^{19}$.

La Autoridad Sanitaria Animal ha determinado que el proyecto entre en una etapa final, denominado "Plan Estratégico 2010-2014”, que tiene como objetivo la erradicación de la brucelosis bovina al año 2014. Para ello, cuenta con tres líneas de acción: vigilancia; saneamiento y eliminación de la infección en un rebaño comprometido y prevención de la transmisión entre los predios ${ }^{19}$.

Finalmente, en el año 2010 se determinó una incidencia nacional de 10,5 animales por 10.000 , a raíz de la detección de 2.690 bovinos serológicamente positivos, los cuales fueron enviados inmediatamente a mataderos. Luego, la prevalencia calculada de acuerdo a los censos serológicos en diciembre del año 2010, fue de 6,4 por 10.000 animales $^{20}$.

Se ha de destacar que las medidas de eliminación y erradicación de la brucelosis bovina afectan directamente los niveles de incidencia de esta zoonosis en la población humana.

En Chile, la brucelosis humana es una enfermedad de notificación obligatoria (ENO) de acuerdo al Decreto Supremo $N^{\circ} 712$ (año 2000) y ratificada en el posterior Decreto Supremo N ${ }^{\circ} 158$ (año 2004). El objetivo del sistema de vigilancia es caracterizar epidemiológicamente los casos, forma de presentación y fuentes de infección, de manera de interrumpir la transmisión y desarrollar medidas de prevención y control en humanos ${ }^{2}$.

De acuerdo a la Norma Técnica $N^{\circ} 55$, sobre Vigilancia de Enfermedades Transmisibles, el caso confirmado co- rresponde al caso sospechoso confirmado por laboratorio y el caso sospechoso es aquel caso compatible con la descripción clínica y vinculado epidemiológicamente a casos presuntos o confirmados en animales o a productos de origen animal contaminados ${ }^{16}$.

El sistema ENO establece la obligación de notificar inmediatamente la sospecha de brucelosis a la Autoridad Sanitaria Regional.

Objetivo del presente estudio: Caracterizar epidemiológicamente la brucelosis humana en Chile.

\section{Métodos}

Se realizó un estudio descriptivo, donde se emplearon datos secundarios provenientes del Sistema ENO del MINSAL, entre 2001-2010 (21). Se distinguieron según la Clasificación Internacional de Enfermedades (CIE10) los códigos A23.0 (brucelosis debida a B. melitensis); A23.1 (brucelosis debida a B. abortus); A23.2 (brucelosis debida a B. suis); A23.3 (brucelosis debida a B. canis); A23.8 (otras brucelosis); A23.9 (brucelosis, no especificada) ${ }^{22}$.

También se utilizaron los datos de Laboratorio, procedentes del Instituto de Salud Pública (ISP). Sólo fue posible obtener información desde el año $2007^{23}$. A la vez, se solicitó al MINSAL los datos de egresos hospitalarios ${ }^{24}$ y el Registro de Mortalidad Nacional ${ }^{25}$. Para ambos se dispuso de datos del período analizado 2001-2010.

En los casos ENO, egresos hospitalarios y defunciones, se calcularon las tasas por año y región, utilizando la población registrada en el Instituto Nacional de Estadísticas-INE ${ }^{26}$.

Mediante estadísticos descriptivos se caracterizó a las personas (sexo, edad, diagnóstico, condición de egreso, nivel de educación, ocupación).

A la vez, se evalúo la variación temporal y geográfica de las tasas de incidencia ENO, egresos hospitalarios y defunciones.

\section{Resultados}

\section{Vigilancia ENO}

En el período 2001-2010 se notifican 90 casos de brucelosis (mediana de 9 casos); 67,8\% correspondió al sexo masculino. El número de casos por año varió de 12 en el año 2001 (58,3\% sexo masculino) a 5 en el año 2010 (60\% sexo masculino).

En el mismo período, la tasa de notificación se ha desplazado desde 0,08 casos por 100 mil habts. a 0,03 casos por 100 mil habts., observándose una tendencia al decrecimiento entre los años 2001 y 2007; en este último año se registró el valor más bajo ( 1 caso, tasa de 0,01 casos por 100.000 habts.). Esta tendencia fue interrumpida en el año 2009, año en que se alcanzó una tasa de 0,09 casos 
Figura 1. Distribución de casos notificados y tasas de brucelosis humana (ENO). Chile, 2001-2010.
Figura 2. Clasificación diagnóstica según CIE10 de los casos de brucelosis humana (ENO). Chile, 2001-2010. A23.0: brucelosis por B. melitensis. A23.1: por B. abortus. A23.2: por B. suis. A23.3: por B. canis. A23.8: otras brucelosis. A23.9: brucelosis, no especificada.

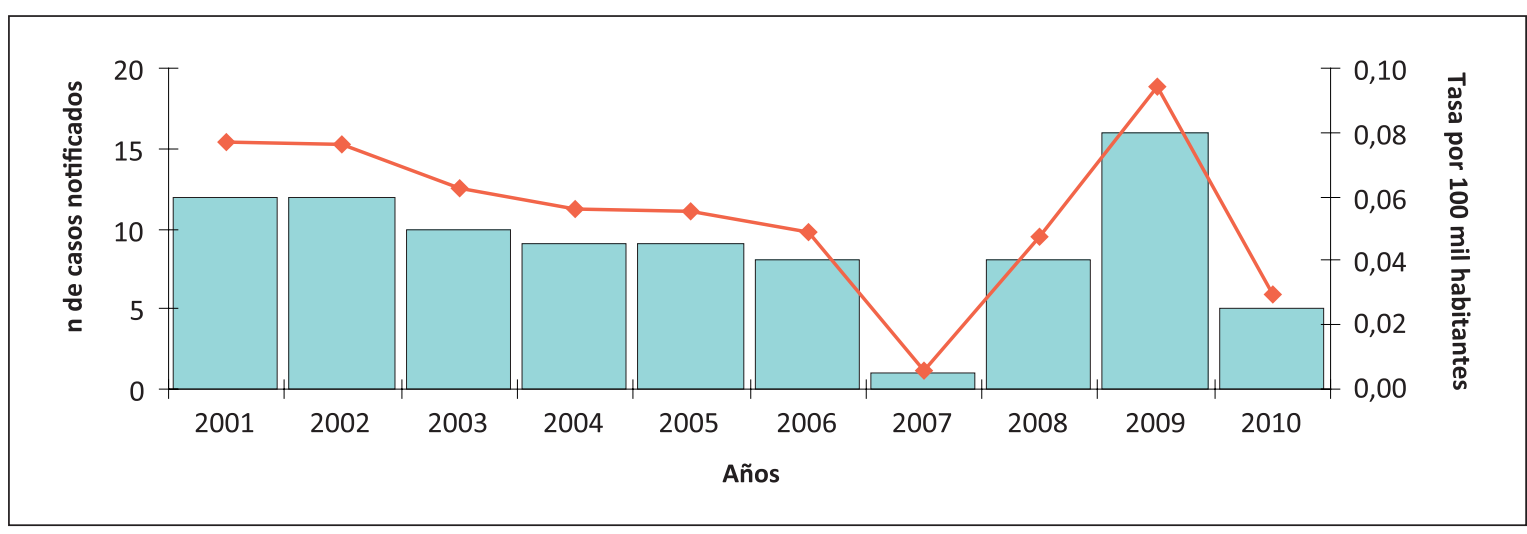

por 100.000 habts, para luego disminuir nuevamente en el año 2010 (Figura 1).

La edad mediana de notificación para el período fue 44 años (rango 1-79 años); hombres 45 años (rango 8-75 años); mujeres 34,5 años (rango 1-79 años).

Según la Clasificación CIE10, el diagnóstico correspondió en $77 \%$ de los casos al código A23.9 y 16\% al A23.1 (Figura 2).

Según distribución geográfica, la mayor tasa de incidencia se registró en la Región de Los Lagos (3,67 casos por 100.000 habts), seguida de la Región de Magallanes (1,92 casos por 100.000 habts.), Región Metropolitana $(0,67$ casos por 100.000 habts.) y Región del Maule $(0,41$ casos por 100.000 habts.). Las tres primeras regiones presentaron tasas superiores a las observadas en el país (0,55 casos por 100.000 habts.) (Figura 3$)$.

\section{Egresos hospitalarios}

Se registran 70 egresos hospitalarios por brucelosis humana para el período 2001-2010. El 68,6\% correspondió
Figura 3. Variación geográfica de los casos y tasas de incidencia de brucelosis humana (ENO). Chile, 2001-2010.
A230 $\square$ A231 $\square$ A232 $\square$ A233 $\square$ A238 $\square$ A239 


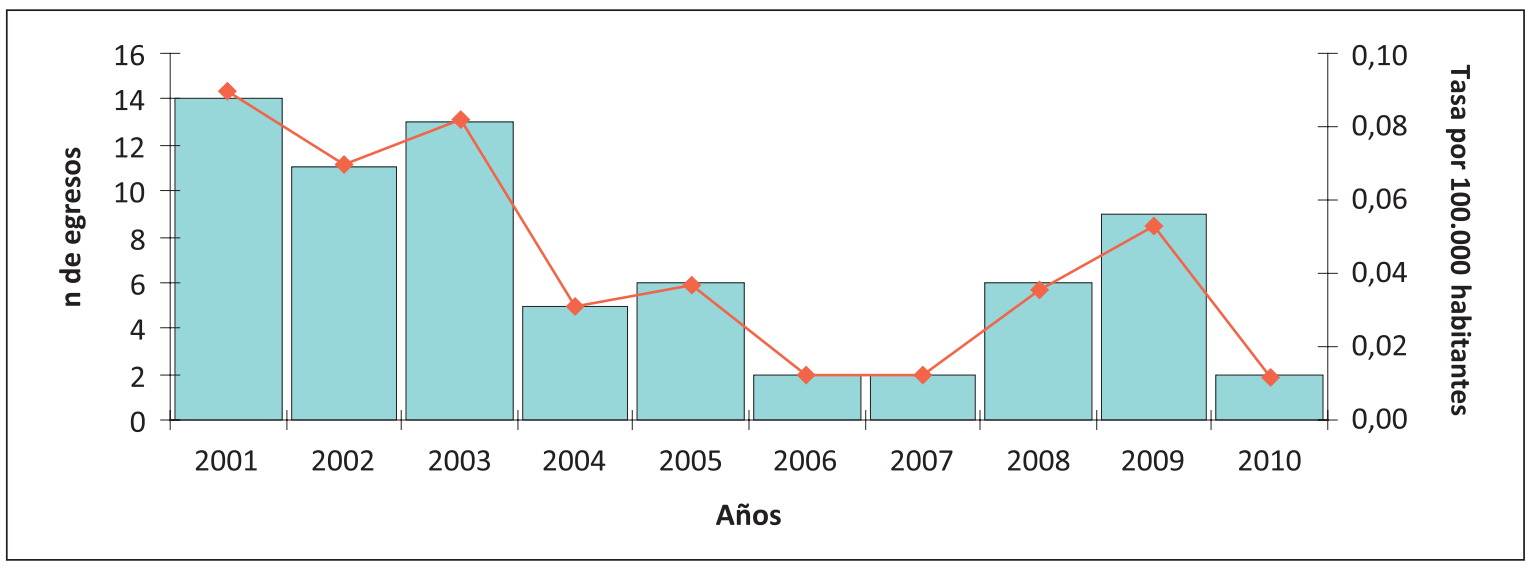

al sexo masculino y su variación fue de 14 para el año 2001 (50\% sexo masculino) a 2 en el año 2010 (50\% sexo masculino), con una mediana de 6 casos. El mayor número de egresos se registró en el año 2001 (14 egresos). La tasa de egresos hospitalarios ha disminuido desde 0,09 en el año 2001 a 0,01 por 100 mil habts., en el año 2010, con tendencia al decrecimiento hasta los años 2006-2007. En los dos años siguientes se observó una tendencia al alza, para volver a descender en el año 2010 (Figura 4).

La edad mediana de los egresos hospitalarios correspondió a 40 años de edad (rango 6 a 77) sin diferencias significativas entre sexos (datos no expuestos).

Según el tiempo de estadía, se registra una mediana de 8 días (rango 1 a 71).

Según diagnóstico CIE10, 84,3\% de los egresos fueron clasificados como A23.9 y 10\% como A23.0. Según su condición al egreso, 92,9\% logró su recuperación.

Según la distribución geográfica, la tasa de egresos de la Región de Magallanes (3,84 por 100.000 habts.), se ubicó sobre la tasa nacional ( 0,43 por 100.000 habts.). Le siguieron Los Lagos (1,27 por 100.000 habts.), Los Ríos (1,07 por 100.000 habts), Aysén (1 por 100.000 habts.), Araucanía (0,54 por 100.000 habts.) y Región Metropolitana ( 0,47 por 100.000 habts.)

\section{Mortalidad}

En el período 2001-2010 se registraron dos defunciones por brucelosis, ambas correspondieron a pacientes de sexo femenino y su mediana de edad fue de 48,5 años (21 y 76 años). La tasa de mortalidad para esos años fue de 0,01 fallecidos por 100.000 habts., y según su residencia, correspondieron a las Regiones de Tarapacá (año 2001) y Metropolitana (año 2009), respectivamente. Según los datos recogidos desde los certificados de defunción, el nivel de instrucción de estas dos personas, correspondió a nivel medio y básico respectivamente y según estado civil, ambas eran solteras, y una en actividad laboral (labores de casa). La segunda pertenecía al sector pasivo (jubilada)

\section{Vigilancia integrada ENO-ISP-Egresos hospitalarios}

La Tabla 1 muestra el número de casos notificados por ENO, los aislamientos confirmados en el ISP y los egresos hospitalarios y defunciones según datos oficiales obtenidos del Registro Civil.

El registro total de egresos hospitalarios (n: 70) es menor que los casos notificados mediante ENO (n: 90). A la vez, los casos confirmados en el ISP (n: 4) son menores a los notificados mediante ENO y a los registros de egresos hospitalarios.

Las cifras de aislados confirmados por ISP y los egresos hospitalarios registrados tuvieron concordancia sólo en el año 2010.

\section{Tabla 1. Casos de brucelosis notificados por ENO, aislados en ISP, egresos} hospitalarios, muertes hospitalarias y defunciones. Chile, 2001-2010

\begin{tabular}{|cccccc}
\hline Años & ENO & ISP & $\begin{array}{c}\text { Egresos } \\
\text { hospitalarios }\end{array}$ & $\begin{array}{c}\text { Muertes } \\
\text { en egresos } \\
\text { hospitalarios }\end{array}$ & Defunciones \\
\hline 2001 & 12 & $\mathrm{~s} / \mathrm{i}$ & 14 & 3 & 1 \\
\hline 2002 & 12 & $\mathrm{~s} / \mathrm{i}$ & 11 & 2 & 0 \\
\hline 2003 & 10 & $\mathrm{~s} / \mathrm{i}$ & 13 & 0 & 0 \\
\hline 2004 & 9 & $\mathrm{~s} / \mathrm{i}$ & 5 & 0 & 0 \\
\hline 2005 & 9 & $\mathrm{~s} / \mathrm{i}$ & 6 & 0 & 0 \\
\hline 2006 & 8 & $\mathrm{~s} / \mathrm{i}$ & 2 & 0 & 0 \\
\hline 2007 & 1 & 0 & 2 & 0 & 0 \\
\hline 2008 & 8 & 2 & 6 & 0 & 0 \\
\hline 2009 & 16 & 0 & 9 & 0 & 1 \\
\hline 2010 & 5 & 2 & 2 & 0 & 0 \\
\hline Total & 90 & 4 & 70 & 5 & 2 \\
\hline
\end{tabular}

$s / i=\sin$ información. ENO: Formulario de Enfermedades de Notificación Obligatoria. ISP: Instituto de Salud Pública. 
La diferencia entre los casos notificados mediante ENO y el número de egresos hospitalarios es de 20 casos. Se discute más adelante la razón que lo explica.

Al comparar el número de defunciones de los pacientes hospitalizados y el número de defunciones específicas obtenidas del Registro Nacional de Defunciones, se observó que no existe correspondencia entre ambos sistemas. El primer sistema registra dos muertes más en el año 2001 y dos defunciones adicionales en el 2002 en relación al Registro Nacional de Mortalidad.

\section{Discusión}

El sexo masculino es el más afectado por la brucelosis, concentrando 67,8 y $68,6 \%$ de los casos en el sistema ENO y en el registro de Egresos Hospitalarios, respectivamente.

Según edad de los casos y de los registros revisados, la edad mayoritariamente se ubica en alrededor de los 40 años, es decir, en plena actividad productiva en términos laborales.

Esta enfermedad está asociada-ya ha sido señalado- a importantes pérdidas económicas en términos de salud humana y a nivel de producción animal.

Al observar el total de casos ENO (n: 90) y egresos hospitalarios (n: 70), se visualiza una diferencia que no es discrepante con la realidad, dado que no todos los pacientes requieren ser hospitalizados; en ambas fuentes se observa un mayor diagnóstico de brucelosis no especificadas (76,7 y 84,3\%, respectivamente). Esta situación da cuenta de la necesidad de avanzar hacia la identificación de las especies involucradas mediante técnicas de última generación.

El 7,8\% de los egresos hospitalarios corresponde a $<18$ años, lo que refleja una infección probablemente no asociada al sector laboral/ ocupacional, concordante con la intensidad de los programas de control animal implementados por el Servicio Agrícola Ganadero-SAG, donde el país ha sido declarado libre de B. melitensis y existen avances en algunas regiones en relación a la brucelosis bovina.

Si bien tanto los casos notificados por ENO como los egresos hospitalarios presentan una tendencia decreciente, se desconoce el número real de casos anuales en el país, lo cual podría ser estimado mediante estudios de prevalencia serológica.

La región con mayor tasa de notificación por ENO y egresos hospitalarios es Magallanes, seguida de la Región de Los Lagos, situación concordante con las zonas de mayor ganadería ovina y bovina del país. Sin embargo, los casos notificados en las personas, no corresponden a enfermedades asociadas al ámbito laboral

De acuerdo al análisis de la mortalidad, llama la atención que las defunciones ocurrieron en personas del sexo femenino, no teniendo antecedentes de sus posibles fuentes de infección. Adicionalmente destaca la no concordancia entre los datos registrados en la base de datos de defunciones del MINSAL y lo registrado en la "Circular de Vigilancia Epidemiológica de Brucelosis" publicada en enero de 2011, donde se señala un sub- registro de muertes asociadas a esta causa desde el año 1990.

De forma anexa, al observar la proporción de muertes en egresos hospitalarios se aprecia una tendencia al decrecimiento, lo cual concuerda con los avances en el diagnóstico y tratamiento; no obstante, llama la atención la no correspondencia con los datos oficiales de defunciones.

Del análisis de estas diferentes fuentes de información, podemos señalar que el sistema de vigilancia epidemiológica debe ser sujeto a una evaluación en términos de consistencia, exactitud, comparabilidad y validez de la información.

También, es relevante mencionar la falta de datos desde el Laboratorio Nacional de Referencia -ISP, encargado de confirmar los casos notificados, el cual sólo dispone de datos desde el año 2007 a pesar que la legislación entregó esta función, a lo menos desde el año 2000, como consta en las Normas Técnicas de Vigilancia de Enfermedades Transmisibles ${ }^{16}$.

Los casos de esta enfermedad y las defunciones por esta patología son evitables, haciéndose necesario profundizar el programa de eliminación y erradicación de la enfermedad animal, la cual incide directamente en los casos en el hombre. Por lo tanto, dado que el SAG ha intensificado la erradicación de la enfermedad bovina para el próximo año (2014), habrá que realizar una evaluación posterior a esta fecha de manera de verificar la concordancia entre abolición de casos bovinos y la disminución y/o eliminación de casos en las personas

\section{Resumen}

Introducción: La brucelosis es una zoonosis con importante impacto económico en las naciones donde es endémica. En Chile su magnitud en medicina humana es poco conocida, de allí que fuera declarada una enfermedad de notificación obligatoria en el año 2004. Objetivo: Caracterizar epidemiológicamente la brucelosis humana en Chile. Material: Se utilizaron datos del Sistema de Notificación Obligatoria, diagnóstico de laboratorio del Instituto de Salud Pública (ISP), egresos hospitalarios y defunciones. Resultados: La incidencia promedio, según las notificaciones obligatorias fue 0,55 casos por 100 mil habs., (hombres 67,8\%, edad mediana 44 años). Los egresos hospitalarios presentaron una tasa promedio de 0,43 egresos por 100 mil habts. El diagnóstico mayormente anotado (84,3\%) fue A23.9 (brucelosis, no especificada). La tasa de mortalidad para 
el período fue de 0,01 defunciones por 100 mil habts., (dos casos). El nivel de instrucción de los fallecidos fue básico. Conclusiones: Las tasas de incidencia de casos notificados, egresos hospitalarios y mortalidad tienden a disminuir. Llama la atención la inconsistencia entre los casos notificados y los aislamientos reportados por el ISP, laboratorio nacional de referencia en esta materia, lo cual sugiere sub-notificación. Es necesario potenciar el sistema de vigilancia caso a caso y optimizar su confirmación por el laboratorio nacional de referencia.

\section{Referencias bibliográfícas}

1.- Seleem M, Boyle S, Sriranganathan N. Brucellosis: A re-emerging zoonosis. Vet Microbiol 2010; 140: 392-8.

2.- Gobierno de Chile. Ministerio de Salud. Reglamento sobre notificación de enfermedades transmisibles de declaración obligatoria $\mathrm{N}^{\mathrm{o}} 158$. Santiago, 22 de octubre de 2004.

3.- Nymo I, Tryland M, Godfroid J. A review of Brucella infection in marine mammals, with special emphasis on Brucella pinnipedialis in the hooded seal (Cystophora cristata). Vet Res 2011; 42: $93<\mathrm{http}: / /$ www.veterinaryresearch. org/content/42/1/93> [Consultado: 19 diciembre de 2012].

4.- Gobierno de Chile. Ministerio de Salud. Circular de Vigilancia epidemiológica de Brucelosis. Enero 2011.

5.- Tiller R, Gee J, Lonsway D, Gribble S, Bell $\mathrm{S}$, Jennsion A, et al. Identification of an unusual Brucella strain (BO2) from a lung biopsy in a 52 year-old patient with chronic destructive pneumonia. BMC Microbiol 2010; 10: $23<\mathrm{http}: / /$ www.biomedcentral.com/14712180/10/23> [Consultado: 19 diciembre de 2012]

6.- Iowa State University. College of Veterinary Medicine. Center for Food Security and Public Health. Institute for International Cooperation in Animal Biologics. "Brucellosis". Julio 2009. 15 pp <http://www.cfsph.iastate.edu/Factsheets/es/ brucelosis.pdf $>$ [Consultado: 26 noviembre de 2012]

7.- Dean A, Crump L, Greter H, Schelling E, Zinsstag J. Global burden of human brucellosis: a systematic review of disease frequency. PLOS Neglec Trop Dis 2012; 6 (10): 1-9.

8.- Atluri V, Xavier M, de Jong M D, den Hartigh A B, Tsolis R. Interactions of the human pathogenic Brucella species with their hosts. Ann Rev Microbiol 2011; 65: 523-41.
9.- Ministerio del Trabajo y Previsión Social; Subsecretaria de Previsión Social. Establece Normas sobre Accidentes del Trabajo y Enfermedades Profesionales. Ley 16.744 de octubre $2011<$ http://www.leychile.cl/ Navegar?idNorma $=28650>$ [Consultado: 13 enero de 2013]

10.- Karsen H, Tekin S, Duygu F, Yapici K, Kati M. Review of 17 cases of neurobrucellosis: clinical manifestations, diagnosis, and management. Arch Iran Med 2012; 15 (8): 491-4.

11.- Ghasemi R, Meraat H, Pahlevan A. A review on ophthalmic manifestations of brucellosis and reporting a case of ophthalmic brucellosis. Iranian Red Crescent Med J 2011; 13 (5): 352-3.

12.- He Y. Analyses of Brucella pathogenesis, host immunity, and vaccine targets using systems biology and bioinformatics. Front Cell Infect Microbiol 2012; 2 (2): 1-17.

13.- Karaali Z, Baysal B, Poturoglu S, Kendir M. Cutaneous manifestations in brucellosis. Indian J Dermatol 2011; 56 (3): 339-40.

14.- Al Dahouk S and Nöckler K. Implications of laboratory diagnosis on brucellosis therapy. Expert Rev Anti-Infect Ther 2001; 9 (7): 833-45.

15.- Christopher S, Umapathy B, Ravikumar K Brucellosis: review on the recent trends in pathogenicity and laboratory diagnosis. J Labor Phys 2010; 2(2): 55-60.

16.- Gobierno de Chile. Ministerio de Salud. Normas Técnicas de Vigilancia de Enfermedades Transmisibles. 2000.

17.- Bems D, Rager B. Emerging infectious diseases: a cause for concern. Israel Med Assoc J 2000; 2 : 919-23.

18.- Gobierno de Chile. Ministerio de Agricultura. Servicio Agrícola y Ganadero. 2012. $<$ http://www.sag.cl/ambitos-de-accion/ brucelosis-bovina-bb $>$ [Consultado: 13 enero de 2013].
19.- Gobierno de Chile. Ministerio de Agricultura. Servicio Agrícola y Ganadero. Subdepartamento de Vigilancia Epidemiológica. División de Protección Pecuaria. Etapas y Resultados de los Proyectos de Control y Erradicación de Brucelosis Bovina, 1975-2006. pp 11. $<$ http://www.sag.cl/OpenDocs/asp/pagDefault. asp?boton $=$ Doc $49 \&$ arg InstanciaId $=49 \& \arg C$ arpetaId $=401 \& \arg$ TreeNodosAbiertos $=(401)$ $(-49) \& \arg$ TreeNodoActual $=401 \& \arg$ TreeNodoS $\mathrm{el}=2>$ [Consultado: 13 enero de 2013] .

20.- Gobierno de Chile. Ministerio de Agricultura. Servicio Agrícola y Ganadero. Subdepartamento de Vigilancia Epidemiológica División de Protección Pecuaria. "Situación Sanitaria de Brucelosis Bovina. 2010". <http://www.sag. cl/ambitos-de-accion/brucelosis-bovina-bb $>$ [Consultado: 13 enero de 2013]

21.- Gobierno de Chile. Ministerio de Salud. Base de datos Enfermedades de Notificación Obligatoria (ENO) 2001-2010.

22.- Organización Panamericana de la Salud (OPS). Clasificación Estadística Internacional de Enfermedades y Problemas relacionados con la Salud. Décima revisión. 1995

23.- Gobierno de Chile. Ministerio de Salud. Instituto de Salud Pública de Chile (ISP). Base de datos de Laboratorio. Brucelosis 2007-2010.

24.- Gobierno de Chile. Ministerio de Salud. Base de datos Egresos Hospitalarios 2001-2010.

25.- Gobierno de Chile. Ministerio de Salud. Base de datos Defunciones 2001-2010.

26.- Gobierno de Chile. Instituto Nacional de Estadística (INE). Departamento de Demografía. Programa de proyecciones de la población. Proyecciones de población por grupos de edad y sexo. $<$ http://www.ine.cl/ canales/chile_estadistico/demografia_y_vitales/ proyecciones/MenPrincOK.xls $>$ [Consultado: 20 diciembre de 2012]. 